\title{
Photocontrol of Supramolecular Azo-Containing Block Copolymer Thin Films During Dip-Coating: Toward Nanoscale Patterned Coatings
}

\author{
Jaana Vapaavuori, ${ }^{\#, a}$ Josué Grosrenaud, ${ }^{\#, a}$ Antti Siiskonen, ${ }^{\mathrm{b}}$ Arri Priimagi, \\ Christian Pellerin*,a and C. Geraldine Bazuin*,a \\ a Département de chimie, Université de Montréal, C.P. 6128, Succursale Centre-Ville, Montréal, \\ QC, Canada H3C 3J7 \\ ${ }^{\mathrm{b}}$ Department of Chemistry and Bioengineering, Tampere University of Technology, \\ Korkeakoulunkatu 8, FI-33101 Tampere, Finland
}

For Table of Contents use only:

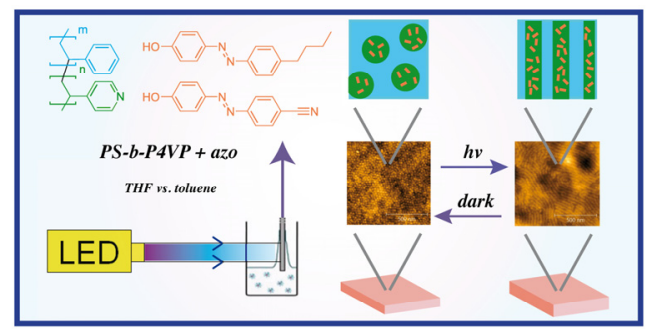


ABSTRACT: Dip-coating allows nanostructured block copolymer (BCP) thin film fabrication in a fast and facile one-step process. It can also be coupled with external controls, such as illumination. Herein, we expose several design principles that enable photocontrol of the nanostructured surface pattern and thickness of supramolecular BCP thin films. This is done using a polystyrene-poly(4-vinylpyridine) (PS-P4VP) BCP and two hydroxy-functionalized smallmolecule (SM) azo derivatives that have different photochemical characteristics and that hydrogen bond to the P4VP block. We show how the film preparation concept provides tunability through the chemical structure of the photoactive SM, the relative amount of SM in the dip-coating solution, and the choice of solvent. It was found that the film thickness and SM uptake in the films are increased by illumination when using THF, but are unchanged when using toluene as solvent, which is attributed to an optical heating effect observable with volatile solvents. The photocontrol of surface patterns is a result of photoinduced changes in the effective volume fraction of the $\mathrm{P} 4 \mathrm{VP}+\mathrm{SM}$ phase, which is increased by greater volume of cis isomers, by higher SM uptake (using THF), and by more trans-cis-trans cycling for systems with shorter cis lifetime. The extent of photoinduced change can also be increased by higher molecular mobility due to more flexible SMs, lower BCP molecular weight, and non-micellar or softer micellar solutions.

Keywords: photocontrol, block copolymer thin films, supramolecular, azo-containing, dipcoating, nanoscale patterns 


\section{INTRODUCTION}

Precise external non-contact control over nanostructured morphologies in thin films of good surface quality is a prerequisite for many applications, such as lithography templates for the electronics industry, ${ }^{1-3}$ platforms for assembling metal nanostructures ${ }^{4,5}$ or proteins, ${ }^{6}$ precursors for nanoporous materials, ${ }^{7}$ and templates for $3 \mathrm{D}$ device nanofabrication. ${ }^{8}$ Block copolymers (BCPs), with their ability to self-assemble spontaneously into various morphologies at length scales from a few to hundreds of nanometers, depending on the block volume fractions, molecular weights and interaction parameters, ${ }^{9}$ are promising candidates for such templates. ${ }^{9-15}$

A highly versatile approach that facilitates the achievement of desired nanopatterns in BCP films is the introduction of guest small molecules (SM) that are block selective via supramolecular interactions such as hydrogen $(\mathrm{H})$ bonding. This allows control over block volume fractions by physical rather than synthetic means. ${ }^{16-21}$ Moreover, dip-coating is an industrially scalable film preparation method that can produce BCP nanopatterns in a single-step process. ${ }^{22-26}$ When combined with the supramolecular approach, it allows dynamic control over the amount of guest SM taken up in the film and therefore over the film morphology. ${ }^{25-27} \mathrm{In}$ addition, it is amenable to simultaneous external stimuli, such as light and temperature, to further control the film structure. In a recent communication, we demonstrated that the thin film surface nanopattern, SM content, and thickness can be modified by visible light illumination of a film during slow dip-coating from a supramolecular BCP solution containing a photoactive azo-based guest SM. ${ }^{28}$ Since light provides very precise spatial and temporal control, this in-situ photocontrol points to pathways for producing complex spatially varying surface patterns.

To understand the physics behind the photoinduced changes in the dip-coated films, several unique features related to dip-coating must be introduced. First, the film thickness has a V-shaped dependence on the withdrawal rate of the substrate from the dip-coating solution (also referred to as the dip-coating rate), as shown for sol-gel films, ${ }^{24}$ block copolymers, ${ }^{25,26}$ and organic semiconductors. ${ }^{29}$ The slow dip-coating rate side of the $\mathrm{V}$ shape, only investigated in recent years, corresponds to the "capillary regime" where the solvent evaporation rate is faster than dipcoating rate, whereas the fast dip-coating rate side corresponds to the long-known "draining regime". ${ }^{23-26}$ Second, we have shown, when dip-coating from supramolecular polystyrene- $b$ poly(4-vinylpyridine) (PS-P4VP) solutions containing either naphthol (NOH) or naphthoic acid 
$(\mathrm{NCOOH})$, both of which hydrogen bond to P4VP, that the molar ratio of SM taken up in the dipcoated film relative to the repeat unit of the preferred block (vinylpyridine or VP), referred to as the SM/VP uptake ratio, varies with dip-coating rate. ${ }^{25-27}$ When using solvents such as tetrahydrofuran (THF) that can compete with P4VP as H-bond acceptors, the uptake ratio is very low at the lowest dip-coating rates and increases with rate throughout the capillary regime, approaching the solution SM/VP molar ratio in the draining regime. ${ }^{25-27}$ With solvents such as toluene that are not H-bond acceptors, the uptake ratios start at a much higher level at low dipcoating rates, so that the tendency to increase with dip-coating rate is much shallower. ${ }^{26}$ The rise in SM/VP uptake ratio as a function of dip-coating rate increases the effective P4VP/PS volume ratio as a function of dip-coating rate, which, in turn, can lead to a sequence of surface nanopatterns, reflecting different (kinetically trapped) film morphologies. ${ }^{25-27,30}$

As a specific example, using PS-P4VP composed of $30 \mathrm{wt} \% \mathrm{P} 4 \mathrm{VP}$ with $\mathrm{NCOOH}$ as the SM and THF as the solvent, the sequence observed starts with a pattern of dots resulting from a spherical morphology, then stripes due to a horizontal cylindrical morphology, followed by dots again resulting this time from vertically oriented cylinders, and finally an islands-and-holes pattern due to terracing by a face-down lamellar morphology. ${ }^{25}$ In contrast, the same BCP/SM system in toluene shows only dot patterns associated with a spherical morphology over the entire dip-coating rate range investigated despite high SM/VP uptake ratios, attributed to immobile (glassy) micellar P4VP cores in solution. ${ }^{26}$

In the above-mentioned communication, the effect of in situ light illumination on dipcoated film characteristics was shown using a similar PS-P4VP BCP combined with a photoresponsive H-bonding azo molecule, 4-butyl-4'-hydroxyazobenzene (BHAB). ${ }^{28}$ This demonstration involved only a single azo SM, BCP, and solvent (THF) in only the slow capillary range of dip-coating. To better comprehend the photoeffects during dip-coating of such supramolecular systems and their potential, it is necessary to investigate the influence of various molecular, environmental and other experimental parameters, and thereby develop guidelines for exploitation and further development of the technique. Here, we expand the scope of the previous communication by investigating the impact on photocontrolled dip-coating of several unstudied aspects. First is the choice of the azo SM used as the photoswitch, where we will compare BHAB with an azo SM with quite different photochemical characteristics, notably 4-cyano-4'hydroxyazobenzene (CHAB). Whereas BHAB has a nonpolar $n$-butyl tail, leading to a long $c i s$ 
lifetime and therefore a quasi-two-state system, $\mathrm{CHAB}$ has an electron-withdrawing and polar cyano tail, leading to faster switching between the trans and cis states. Second is the choice of solvent, notably toluene compared to THF, given the quite different results for the two solvents as mentioned above, which are related to H-bonding or not with the solvent, to the solvent volatility, and to solution micellar characteristics. In addition, the investigations are extended to the faster draining range of dip-coating and to a BCP with higher molecular weight. The molecular structures of the BCP and SMs and the experimental setup are shown in Scheme 1.

Scheme 1. Chemical Structure of the Block Copolymer (BCP) and Azobenzene-Based Small Molecules (SM) Used, and a Schematic of the Experimental Setup.

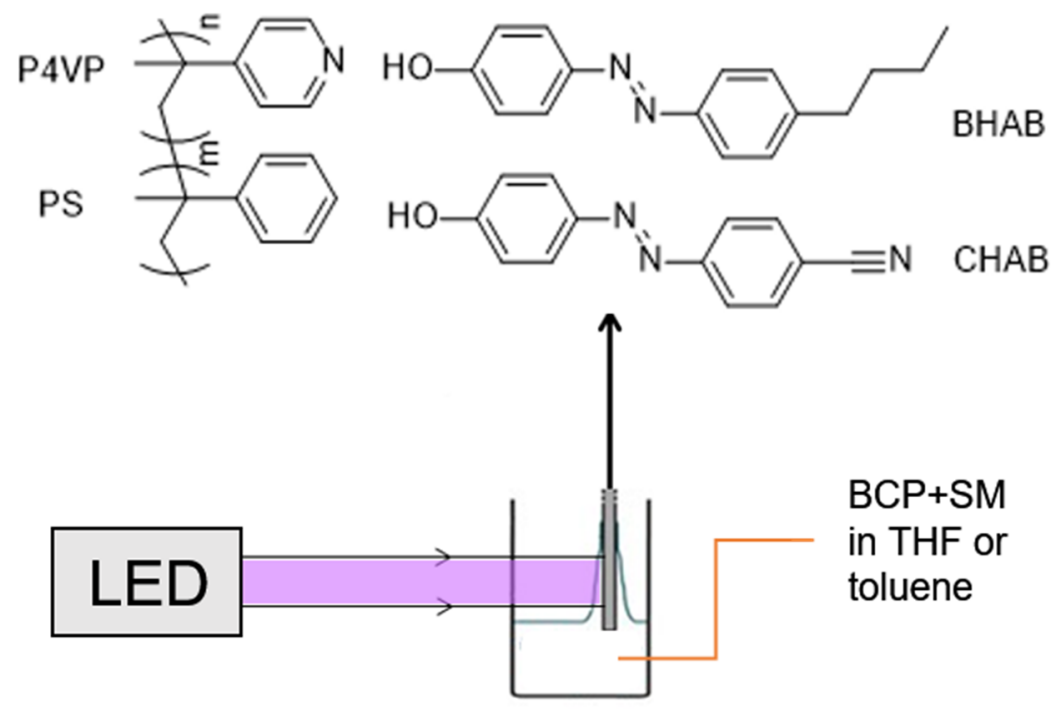

\section{EXPERIMENTAL SECTION}

Materials and Solution Preparation. The PS-P4VP block copolymer [referred to as P; $\left.\mathrm{M}_{\mathrm{n}}(\mathrm{PS})=24,000 \mathrm{~g} / \mathrm{mol}, \mathrm{M}_{\mathrm{n}}(\mathrm{P} 4 \mathrm{VP})=9500 \mathrm{~g} / \mathrm{mol}, \mathrm{PDI}=1.10\right]$, BHAB and CHAB were purchased from Polymer Source, Tokyo Chemical Industries, and Beam Co., respectively, and were used as received. For a comparative study reported in the Supporting Information (SI), a higher molecular weight PS-P4VP [referred to as P'; $\mathrm{M}_{\mathrm{n}}(\mathrm{PS})=36,500 \mathrm{~g} / \mathrm{mol}, \mathrm{M}_{\mathrm{n}}(\mathrm{P} 4 \mathrm{VP})=16,000 \mathrm{~g} / \mathrm{mol}, \mathrm{PDI}=1.15$ ] from Polymer Source was also employed. The blend components were weighed to give the desired 
SM/VP molar ratios and dissolved together in either THF or toluene (Fisher Scientific, purified to 99.9\% under Ar using the Inert PureSolv MD7 solvent purification system) to give solutions of 10 $\mathrm{mg} / \mathrm{mL}$ with respect to the polymer. For comparison, SM-free solutions of the BCPs were prepared at the same concentration. The solutions were stirred for at least $24 \mathrm{~h}$ at room temperature and then filtered through 0.45 and $0.25 \mu \mathrm{m}$ PTFE filters. When not in use, the vials containing the solutions were sealed by parafilm (Pechiney) and kept in the dark. The systems will be referred to by the general nomenclature, $\mathrm{P} / \mathrm{SM}_{\mathrm{x}}$, where $\mathrm{x}$ refers to the $\mathrm{SM} / \mathrm{VP}$ molar ratio in the dip-coating solution.

Solution Characterization by Dynamic Light Scattering (DLS). For DLS, solutions of $0.5 \mathrm{mg} / \mathrm{mL}$ were prepared as above. A Brookhaven BI-200SM instrument equipped with a 532$\mathrm{nm}$ laser at an angle of $90^{\circ}$ was used for the measurements. The solution temperature was controlled with a thermostatic bath set to $20{ }^{\circ} \mathrm{C}$. All reported values are an average of 5 measurements.

Film Preparation by Dip-Coating and Simultaneous Illumination. Silicon wafers $(1,0,0)$, purchased from University Wafer, were used as dip-coating substrates, and, prior to use, were cleaned in a $150{ }^{\circ} \mathrm{C}$ piranha solution for at least $30 \mathrm{~min}$ and then washed with Milli-Q water and blow-dried with nitrogen prior to use. Dip-coating was done in ambient conditions $\left(21 \pm 2{ }^{\circ} \mathrm{C}\right.$; variable relative humidity with no evidence of a significant effect on the results obtained) both in the dark and under illumination, using a KSV-NIMA dip-coater (KN4001). In situ illumination, shown schematically in Scheme 1, was effected by directing a collimated LED light beam (Prizmatix FC5 Multichannel LED light, $365-\mathrm{nm}, 40 \mathrm{~mW} / \mathrm{cm}^{2}$ as measured at the sample location) towards the substrate, centered at a level just above the solution and in parallel to the solution surface, during withdrawal of the substrate from the solution. Both the LED source and the dipcoater were placed on an optical table, with a distance of about $15 \mathrm{~cm}$ between the source and substrate. In all cases, the silicon substrates were immersed vertically into the solution at a rate of $5.0 \mathrm{~mm} / \mathrm{min}$ to a depth of $8 \mathrm{~mm}$, held there for $30 \mathrm{~s}$, and then withdrawn at the desired dip-coating rate. Thus, the time of exposure of the developing film to illumination is shorter the faster the dipcoating rate. The dip-coated films were left to dry overnight in covered containers at room temperature.

Photochemical Characterization. The same illumination conditions as employed for dipcoating were used for measuring the photoresponse of solutions and thin films. Dilute THF 
solutions of pure $\mathrm{BHAB}$ or $\mathrm{CHAB}(0.0085 \mathrm{mg} / \mathrm{mL})$ and of $\mathrm{P} / \mathrm{BHAB}_{0.25}$ or $\mathrm{P} / \mathrm{CHAB}_{0.25}(0.05$ $\mathrm{mg} / \mathrm{mL}$ with respect to the block copolymer) and dilute toluene solutions of pure BHAB (0.017 $\mathrm{mg} / \mathrm{mL})$ and $\mathrm{P} / \mathrm{BHAB}_{0.50}(0.05 \mathrm{mg} / \mathrm{mL}$ with respect to the block copolymer) were irradiated for 5 min, and their UV-visible absorption spectra were recorded by an Ocean Optics 2000+ fibercoupled spectrometer using a DH-mini UV-Vis-NIR light source.

Computations. All calculations were performed with Gaussian 09 Revision D.01 (Gaussian Inc., Wallingford CT, 2009), employing the PBE0/6-311++G(d,p) method. An ultrafine grid was used for all calculations. The solvent was implicitly simulated using the SMD solvent model by Marenich et al. ${ }^{31}$ For the interaction energy calculations, done in vacuo, 4-ethylpyridine was used as a model for the P4VP polymer and the Boys and Bernardi counterpoise correction was applied to correct for the basis set superposition error. The time-dependent DFT method was used to calculate the excitation energies.

Thin Film Characterization. Morphology characterization of the dip-coated films was done with a Bruker Multimode AFM microscope controlled by a Nanoscope V controller. Nanoworld tips (Arrow-NCR, Al-coated, force constant $42 \mathrm{~N} / \mathrm{m}$, optimum frequency $285 \mathrm{kHz}$ ) were used in tapping mode. In many cases, the dried films were rinsed in methanol $(\mathrm{MeOH})$ for at least $30 \mathrm{~s}$ (up to a maximum of $2 \mathrm{~min}$ ). Topographic images were leveled by removing a second degree polynomial background and the height color scale (z-scale) was adjusted to optimize the contrast between the morphological features. Film thicknesses were determined by an EC-400 spectroscopic ellipsometer (J. A. Woollam) at an angle of $45^{\circ}$ by averaging 3 measurements, using the best fits of Cauchy models taking into account the natural $\mathrm{SiO}_{2}$ oxide layer at the surface of the silicon wafers.

The films were further characterized by attenuated total reflection (ATR) infrared spectroscopy (IR) using a Bruker Optics Tensor 27 spectrometer and a hemispherical Ge ATR crystal (variGATR, Harrick Scientific), with p-polarized illumination at an incidence angle of $60^{\circ}$. All spectra were obtained by an accumulation of 512 interferograms at a resolution of $4 \mathrm{~cm}^{-1}$. To examine the BHAB/VP uptake ratio in the films dip-coated from $\mathrm{P} / \mathrm{BHAB}_{0.25}$ (and $\mathrm{P}^{\prime} / \mathrm{BHAB}_{0.25}$ ) solutions, a calibration curve was determined using the $1492 \mathrm{~cm}^{-1} \mathrm{P} 4 \mathrm{VP}$ band and the $1505 \mathrm{~cm}^{-1} \mathrm{BHAB}$ band in films obtained by directly drop-casting four $10 \mathrm{mg} / \mathrm{mL} \mathrm{CHCl}$ solutions with BHAB/VP ratios of $0,0.060,0.12$ and 0.25 onto the Ge ATR crystal (for P', a 
BHAB/VP ratio of 0.18 was added). Due to partial overlap of the 1492 and $1505 \mathrm{~cm}^{-1}$ bands, second derivative intensities of the absorbance spectra were used to calculate band ratios. A second calibration curve, for use with higher molar ratio dip-coating solutions, was established with 8 different $\mathrm{BHAB} / \mathrm{VP}$ ratios up to 1.0, where normal absorbance intensities were used to calculate band ratios. For the $\mathrm{CHAB}$ complexes, 5 calibration solutions up to the $0.25 \mathrm{CHAB} / \mathrm{VP}$ ratio were used. For these, the high intensity and isolation of the $2227 \mathrm{~cm}^{-1}$ CHAB nitrile stretching band allowed the normal absorbance intensities to be used for calculating the band ratios and led to less dispersion in the calibration points than for the BHAB complexes. Each calibration point is an average of three measurements.

\section{RESULTS AND DISCUSSION}

Photochemical Characteristics. Some pertinent photochemical characteristics in solution of the azobenzene derivatives employed, with and without $\mathrm{P}$ present, are summarized in Table 1. The UV-visible spectra of BHAB and CHAB in dilute THF solution as well as BHAB in dilute toluene solution, in the dark and under illumination, are given in Figure 1. Clearly, the difference in the chromophore tail (butyl vs. cyano) has a much greater influence on the absorption maximum in the dark, $\lambda_{\max }$, than does the difference in solvent (THF vs. toluene), the former showing a difference of close to $30 \mathrm{~nm}$ compared to only $4 \mathrm{~nm}$ for the latter. This can be correlated with the difference in their ground state dipole moments, computed to be 1.74 and $8.49 \mathrm{D}$ for BHAB and $\mathrm{CHAB}$, respectively, when using the dielectric constant of THF as the environment, whereas there is only a small reduction to $1.55 \mathrm{D}$ for BHAB when replacing THF by toluene as the implicit solvent. When $\mathrm{P}$ is present in the solution (at the SM/VP molar ratios investigated below), the $\lambda_{\max }$ for BHAB in either solvent hardly changes, giving values of $353 \pm 4 \mathrm{~nm}$ considering both solvents with and without $\mathrm{P}$ present. In contrast, for $\mathrm{P} / \mathrm{CHAB}_{0.25}$ in THF, the value of $\lambda_{\max }$, although still significantly higher than for $\mathrm{P} / \mathrm{BHAB}_{0.25}$, moves to a wavelength that is $13 \mathrm{~nm}$ lower compared to CHAB alone in THF. This suggests that CHAB is more sensitive than BHAB to small changes in its molecular environment, such as those induced by the presence of the copolymer in the solution, possibly because of its greater polarity. Indeed, the dielectric constant of THF must be reduced somewhat by the addition of $\mathrm{P}$, whose majority component, PS, has a much lower dipole moment $(0.29 \mathrm{D})^{32}$ than THF $(1.7 \mathrm{D})^{33}$ does. 
Table 1. Photochemical Characteristics of the BHAB and CHAB Systems Studied

\begin{tabular}{lccc}
\hline Sample & $\boldsymbol{\lambda}_{\max }(\mathbf{n m})$ & cis $(\mathbf{\%})$ & $\mathbf{t}_{\mathbf{1} / \mathbf{2}}(\mathbf{m i n})$ \\
\hline BHAB, THF & 353 & 96 & 53 \\
BHAB, toluene & 349 & 90 & 4.6 \\
CHAB, THF & 380 & 65 & 0.7 \\
P/BHAB 0.25, THF & 353 & 93 & 69 \\
P/BHAB $_{0.50}$, toluene & 356 & 95 & 7.7 \\
P/CHAB $_{0.25}$, THF & 367 & 91 & 1.8 \\
\hline
\end{tabular}

$\lambda_{\text {max }}$ : absorption maximum in the dark; cis: minimum cis content in the photostationary state; $\mathrm{t}_{1 / 2}$ : half-life of the cis isomer

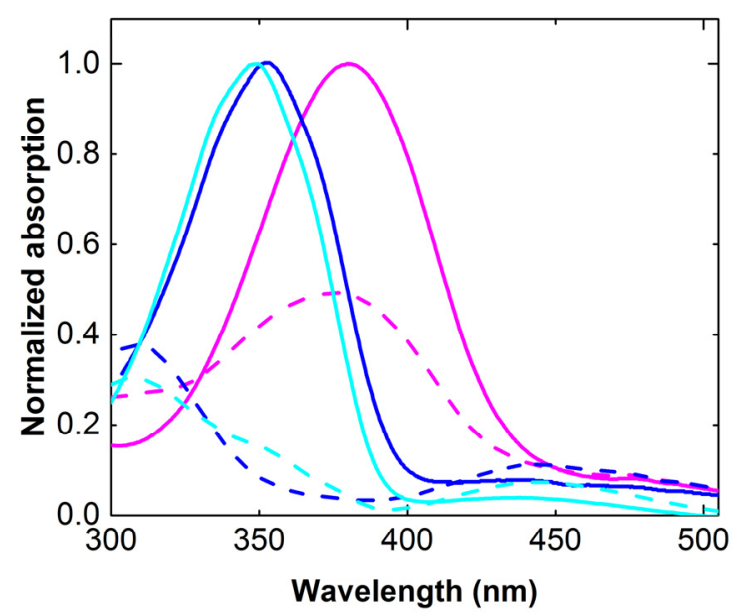

Figure 1. Normalized UV-visible spectra of dilute THF solutions of BHAB (blue) and CHAB (magenta), as well as of a dilute toluene solution of BHAB (cyan), in darkness (solid lines) and under illumination of $40 \mathrm{~mW} / \mathrm{cm}^{2}$ at $365 \mathrm{~nm}$ (dashed lines).

When azobenzenes are illuminated, the predominant photoisomerization reaction can be switched by changing the illumination wavelength. Of the four different LED wavelengths available to us for photoirradiation (ranging from 365 to $520 \mathrm{~nm}$ ), the $365-\mathrm{nm}$ LED at $40 \mathrm{~mW} / \mathrm{cm}^{2}$ was chosen since it gives the highest trans-cis conversion in the photostationary state, notably at least $96 \%$ and $65 \%$ for dilute $\mathrm{BHAB}$ and $\mathrm{CHAB}$ in $\mathrm{THF}$, respectively, as estimated from the ratio 
of the trans absorbance band in the photostationary state to the initial absorbance (Table 1 and Figure 1). This wavelength is close to the $\pi-\pi^{*}$ absorption maximum of the trans isomers (computed values in THF of $370 \mathrm{~nm}$ and $384 \mathrm{~nm}$ for BHAB and CHAB, respectively), with little or no overlap by the absorption of the cis isomers, for which there are two maxima, computed to be at 316 and $479 \mathrm{~nm}$ for BHAB and at 323 and $474 \mathrm{~nm}$ for CHAB. For BHAB, the highly cisrich state under illumination allows measuring the cis $\pi-\pi^{*}$ and $\mathrm{n}-\pi^{*}$ band positions with reasonable accuracy (determined from the dashed spectrum in Figure 1), being 310 and $445 \mathrm{~nm}$, which are fairly close to the computed values. Thus, due to minimal absorption of $c i s$ isomers at $365 \mathrm{~nm}$, mainly photoisomerization from trans to $\mathrm{cis}$ is activated by $365-\mathrm{nm}$ illumination. For $\mathrm{CHAB}$, when $\mathrm{P}$ is added to the solution, the blue shift of the absorption maximum is accompanied by a significant increase in the $c i s$-isomer content (from $65 \%$ to $91 \%$ ). This is due to the $\pi$ - $\pi^{*}$ absorption band being more optimally addressed by $365-\mathrm{nm}$ illumination and to the cis lifetime increasing by addition of $\mathrm{P}$, since the effective dielectric constant of the solution changes. Analogous alteration of the cis lifetime upon addition of PS has been observed for another azobenzene system. ${ }^{32}$

The cis half-lives of BHAB in THF, with and without the presence of $\mathrm{P}$, are much greater than those of CHAB in THF, at around $1 \mathrm{~h}$ versus less than 2 min (Table 1). Due to the very small absorption of BHAB cis molecules at $365 \mathrm{~nm}$, photoinduced cis-trans back isomerization is negligible and the photostationary state essentially results from the equilibrium of the photoinduced trans-cis reaction and the thermal cis-trans back reaction. Since CHAB cis isomers relax thermally much faster than $\mathrm{BHAB}$ cis isomers, the $\mathrm{CHAB}$ molecules must undergo more trans-cis-trans isomerization cycles per time unit than $\mathrm{BHAB}$ molecules to retain the $>90 \%$ cisisomer content observed in the photostationary state. When changing the solvent from THF to toluene for $\mathrm{BHAB}$, the cis lifetimes are shortened as well, contrasting with the tendency observed in the literature to the effect that a decreasing solvent polarity increases the cis lifetime. ${ }^{34,35}$ However, recent research indicates that the influence of solvent on thermal isomerization kinetics is complex, involving not only the solvent polarity but also factors that include the preferred isomerization mechanism, azobenzene substituents, solution concentration, and the presence of intra- and intermolecular hydrogen bonding. ${ }^{36-38}$ In our case, it is noteworthy that toluene does not form hydrogen bonds with the $\mathrm{OH}$-functionalized azos, in contrast to THF, which may account for the shorter cis lifetimes in toluene, thus outweighing the effect of solvent polarity. 
Effects of Azobenzene Type (BHAB vs. CHAB). For reference in what follows in this section and the next, the dip-coated film surface patterns observed by AFM for the systems investigated at the various dip-coating rates used are summarized in Table 2. Representative supporting AFM topographic images for Table 2 are gathered in Section S1 (Figures S1a-h) of the SI. The morphological assignments, explained below, rely in part on what was learned in our earlier work. ${ }^{16,25-28,30}$ In a number of cases shown in the SI and specified in the relevant figure captions, the dried films used for AFM analysis (but not for ellipsometric or infrared analysis)

Table 2. Surface Patterns Observed in AFM Images (Section S1 in the Supporting Information) of Films Dip-Coated in the Dark and Under 365-nm LED Illumination

\begin{tabular}{|c|c|c|c|c|c|c|c|c|c|c|c|}
\hline \multirow{2}{*}{$\begin{array}{l}\text { Dip-coating } \\
\text { solution }\end{array}$} & \multicolumn{11}{|c|}{ Dip-coating withdrawal rate $(\mathrm{mm} / \mathrm{min})$} \\
\hline & 0.3 & 0.5 & 1.0 & 2.0 & 3.0 & 4.0 & 7.0 & 10 & 20 & 40 & 80 \\
\hline $\begin{array}{l}P \text { in THF, } \\
\text { in dark }\end{array}$ & & $\mathrm{d}$ & $\mathrm{d}$ & $\mathrm{d}$ & & $\mathrm{d}$ & $\mathrm{d}$ & $\mathrm{d}$ & $d / s^{*}$ & $\mathrm{~d} / \mathrm{s}^{*}$ & $d\left(\mathrm{~s}^{*}\right)$ \\
\hline $\begin{array}{l}\mathbf{P} / \mathbf{B H A B}_{0.25} \\
\text { in THF, } \\
\text { in dark }\end{array}$ & & d & $\mathrm{d} / \mathrm{s}$ & $\mathrm{s}$ & & $\mathrm{s}$ & $\mathrm{s}$ & $\mathrm{s}$ & $\mathrm{s}$ & $\mathrm{s}(\mathrm{d})$ & $\mathrm{s}(\mathrm{d})$ \\
\hline $\begin{array}{l}\mathrm{P} \mathrm{BHAB}_{0.25} \\
\text { in THF, } \\
\text { under light }\end{array}$ & & $\mathrm{s}$ & $\mathrm{s}$ & s & & $\mathrm{s}$ & $\mathrm{s}$ & $\mathrm{s}$ & $\mathrm{s}$ & $\mathrm{s}(\mathrm{d})$ & $s(d)$ \\
\hline $\begin{array}{l}\mathbf{P} / \mathrm{CHAB}_{0.25} \\
\text { in THF, } \\
\text { in dark }\end{array}$ & & $\mathrm{d}$ & $\mathrm{d}$ & $\mathrm{d}\left(\mathrm{s}^{*}\right)$ & & $\mathrm{d} / \mathrm{s}$ & $\mathrm{s}$ & $\mathrm{s}$ & $\mathrm{s}(\mathrm{d})$ & $\mathrm{d} / \mathrm{s}$ & $\mathrm{d}\left(\mathrm{s}^{*}\right)$ \\
\hline $\begin{array}{l}\mathrm{P} / \mathrm{CHAB}_{0.25} \\
\text { in THF, } \\
\text { under light }\end{array}$ & & $\mathrm{d}(\mathrm{s})$ & $\mathrm{d} / \mathrm{s}$ & $\mathrm{d} / \mathrm{s}$ & & $\mathrm{s}(\mathrm{d})$ & $\mathrm{s}$ & $\mathrm{s}$ & $\mathrm{s}(\mathrm{d})$ & $\mathrm{d} / \mathrm{s}$ & $\mathrm{d}\left(\mathrm{s}^{*}\right)$ \\
\hline $\begin{array}{l}\mathbf{P} \text { in toluene, } \\
\text { in dark }\end{array}$ & $\mathrm{d}$ & $\mathrm{d}$ & $\mathrm{d}$ & $\mathrm{d}$ & $\mathrm{d}$ & & & $\mathrm{d}$ & $\mathrm{d}$ & $\mathrm{d}$ & $\mathrm{d}$ \\
\hline $\begin{array}{l}\mathrm{P} / \mathrm{BHAB}_{0.50} \\
\text { in toluene, } \\
\text { in dark }\end{array}$ & $\mathrm{d}\left(\mathrm{s}^{*}\right)$ & $\mathrm{d}\left(\mathrm{s}^{*}\right)$ & $\mathrm{s}(\mathrm{d})$ & $\mathrm{s}(\mathrm{d})$ & $\mathrm{u}$ & & & $\mathrm{d}$ & $\mathrm{d}$ & $\mathrm{d}$ & $\mathrm{d}$ \\
\hline $\begin{array}{l}\mathrm{P} / \mathrm{BHAB}_{0.50} \\
\text { in toluene, } \\
\text { under light }\end{array}$ & $\mathrm{d} / \mathrm{s}$ & $\mathrm{d} / \mathrm{s}$ & $\mathrm{s}(\mathrm{d})$ & $\mathrm{s}(\mathrm{d})$ & $\mathrm{u}$ & & & $\mathrm{d} / \mathrm{s}^{*}$ & $\mathrm{~d} / \mathrm{s}^{*}$ & $\mathrm{~d} / \mathrm{s}^{*}$ & $\mathrm{~d} / \mathrm{s}^{*}$ \\
\hline
\end{tabular}

P: PS(24k)-P4VP(9.5k); d: dots; s: stripes; $\mathrm{s}^{*}$ : very short stripes; $\mathrm{d} / \mathrm{s}$ : mixed dots and stripes; (): minor component; u: unclear; no experiments done where blank. 
were rinsed briefly in $\mathrm{MeOH}$, which is a selective solvent for P4VP and the SMs and a nonsolvent for PS, to help reveal the morphology. This rinsing results in the original P4VP+SM dots and stripes lying at a lower level than the PS matrix due to washing out of the SM and partial entrainment of P4VP segments on top of the PS, without perturbing the overall morphology due to its maintenance by the glassy PS matrix..$^{16,26,30}$ In one series (last line in Table 2 for the higher dip-coating rates), phase images appear clearer than the topographic images and are thus included in the SI.

It must be kept in mind that dip-coated films are in effect subject to partial solventannealing due to the relatively slow evaporation rate of the solvent (compared, for example, with the much faster rate for spin-coated films). This can allow a certain degree of morphology evolution along a pathway between the initial solution morphology and the equilibrium bulk morphology during solvent evaporation until there is insufficient molecular mobility and therefore kinetic trapping of the morphology achieved at that point. ${ }^{26}$ Thus, the morphologies of dip-coated films are generally less well developed than in classic solvent-annealed films.

As mentioned in the Introduction, our recent communication focused on dip-coating in the capillary regime using the $\mathrm{P} / \mathrm{BHAB}_{0.25}$ system in THF. Figures $2 \mathrm{a}$ and $2 \mathrm{~b}$ and Table 2 extend the results obtained for $\mathrm{P} / \mathrm{BHAB}_{0.25}$ to the draining regime concerning the average film thicknesses, $\mathrm{BHAB} / \mathrm{VP}$ uptake ratios and surface patterns without and with illumination, and compare these data with what is observed when BHAB is replaced by CHAB. In presenting these results, we will first describe the trends for dip-coating without illumination and then the changes caused by illumination.

Without illumination. The average film thicknesses as a function of withdrawal rate for the films dip-coated from THF solutions of pure $\mathrm{P}, \mathrm{P} / \mathrm{BHAB}_{0.25}$ and $\mathrm{P} / \mathrm{CHAB}_{0.25}$ without LED illumination (Figure 2a) follow the expected V-shaped curve, as defined by the capillary and draining regimes. The average thicknesses at any given dip-coating rate, as well as the minimum point in the V-curves, of the BHAB- and CHAB-containing films and the SM-free films are very similar, in accordance with previous findings with other PS-P4VP/SM solutions in THF. ${ }^{25,27}$ 

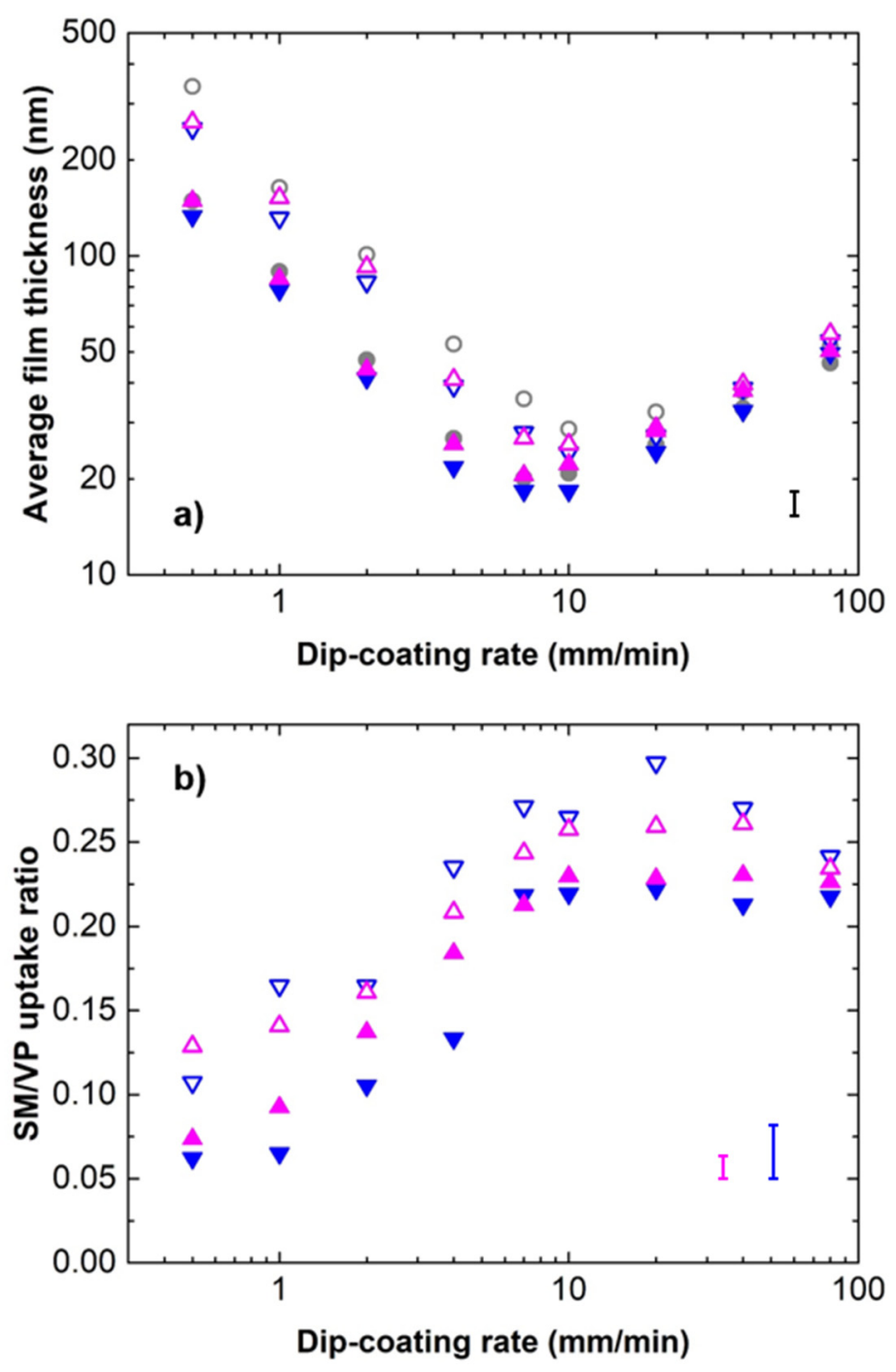

Figure 2. (a) Average film thickness and (b) SM/VP uptake ratio as a function of withdrawal rate for films dip-coated in the dark (closed symbols) and under LED illumination (open symbols) from THF solutions of $\mathrm{P} / \mathrm{BHAB}_{0.25}$ (blue down triangles), $\mathrm{P} / \mathrm{CHAB}_{0.25}$ (magenta up triangles), and, in (a), pure $\mathrm{P}$ (gray circles). Error bars (color-coded in b) are indicated in the lower right corners of the graphs.

The trends in the $\mathrm{BHAB} / \mathrm{VP}$ and $\mathrm{CHAB} / \mathrm{VP}$ uptake ratios are likewise the same as found previously for other P/SM solutions in THF ${ }^{25,27}$ notably, the uptake ratios start out very low relative to that present in solution, increases with increasing dip-coating rate in the capillary regime and approaches the solution ratio in the draining regime (Figure 2b). The low relative uptake ratio at slow dip-coating rates has been attributed to the fact that, by virtue of what defines the capillary 
regime, the drying film is in contact with the solution meniscus and this allows extraction of some fraction of the small molecules back into solution. ${ }^{25,26}$ Consequently, the slower the dip-coating rate, the more time there is for this extraction to occur and the lower the uptake ratio is, particularly in solvents like THF that are H-bond competitors of P4VP.

AFM height images of the films dip-coated from the THF solutions of $\mathrm{P} / \mathrm{BHAB}_{0.25}$ (Figures 3 and $\mathrm{S} 1 \mathrm{~b}$ ) and $\mathrm{P} / \mathrm{CHAB}_{0.25}$ (Figures 4 and $\mathrm{S} 1 \mathrm{~d}$ ) show an evolution in their surface patterns as a function of dip-coating rate that reflects a morphology evolution due to the changing uptake ratio. For the BHAB system, there is a clear transition from dots (d) to stripes (s), indicative of spheres and horizontal cylinders, respectively, in the vicinity of the $1.0 \mathrm{~mm} / \mathrm{min}$ dip-coating rate for the 0.25 molar ratio, as described in ref 28 and indicated in Table 2. The stripe pattern is maintained thereafter, except for the two highest dip-coating rates used, where some dots are mixed with the (still predominant) stripes.

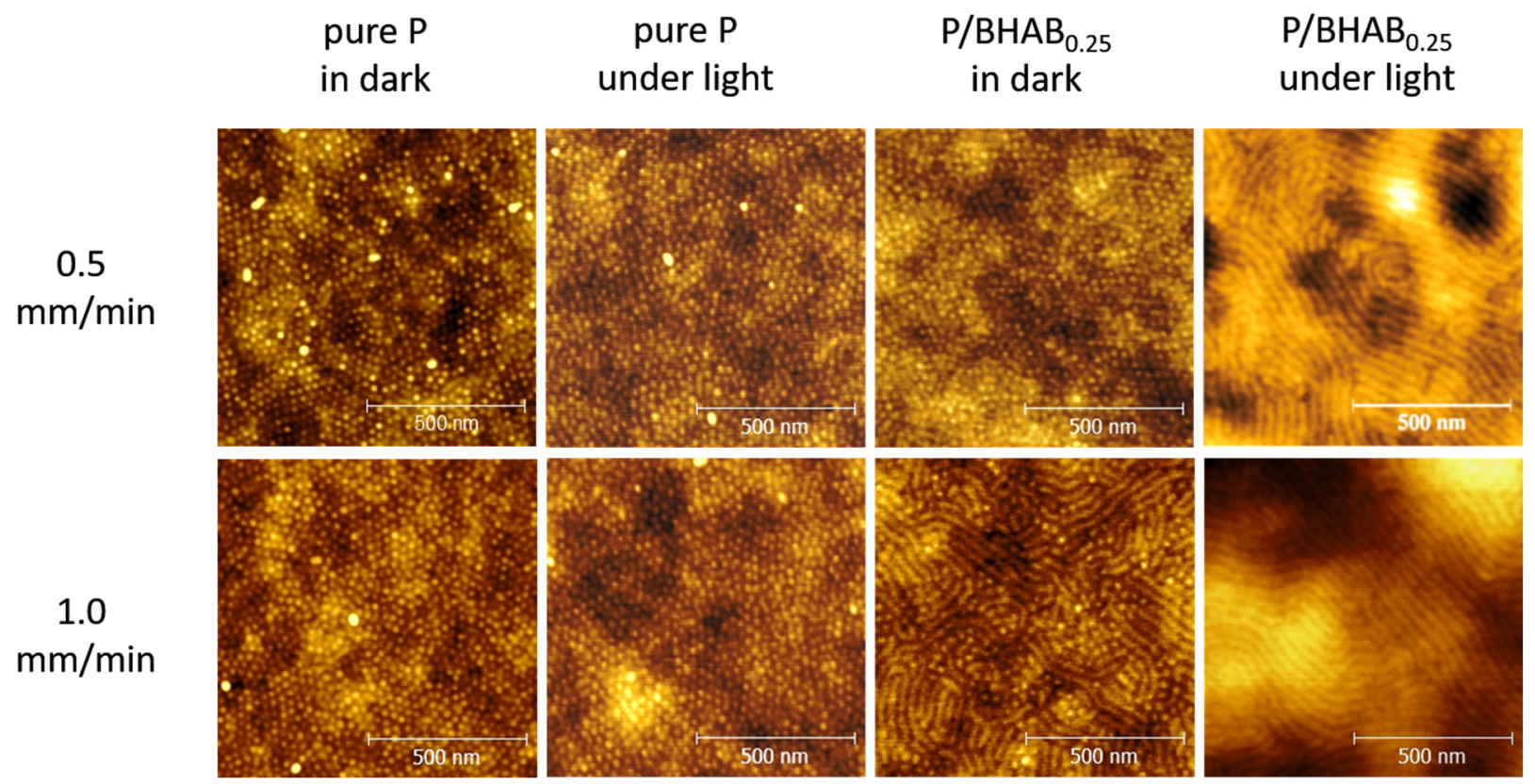

Figure 3. Representative AFM height images of films dip-coated in the dark and under LED illumination from a THF solution of $\mathrm{P} / \mathrm{BHAB}_{0.25}$ at the withdrawal rates indicated. 


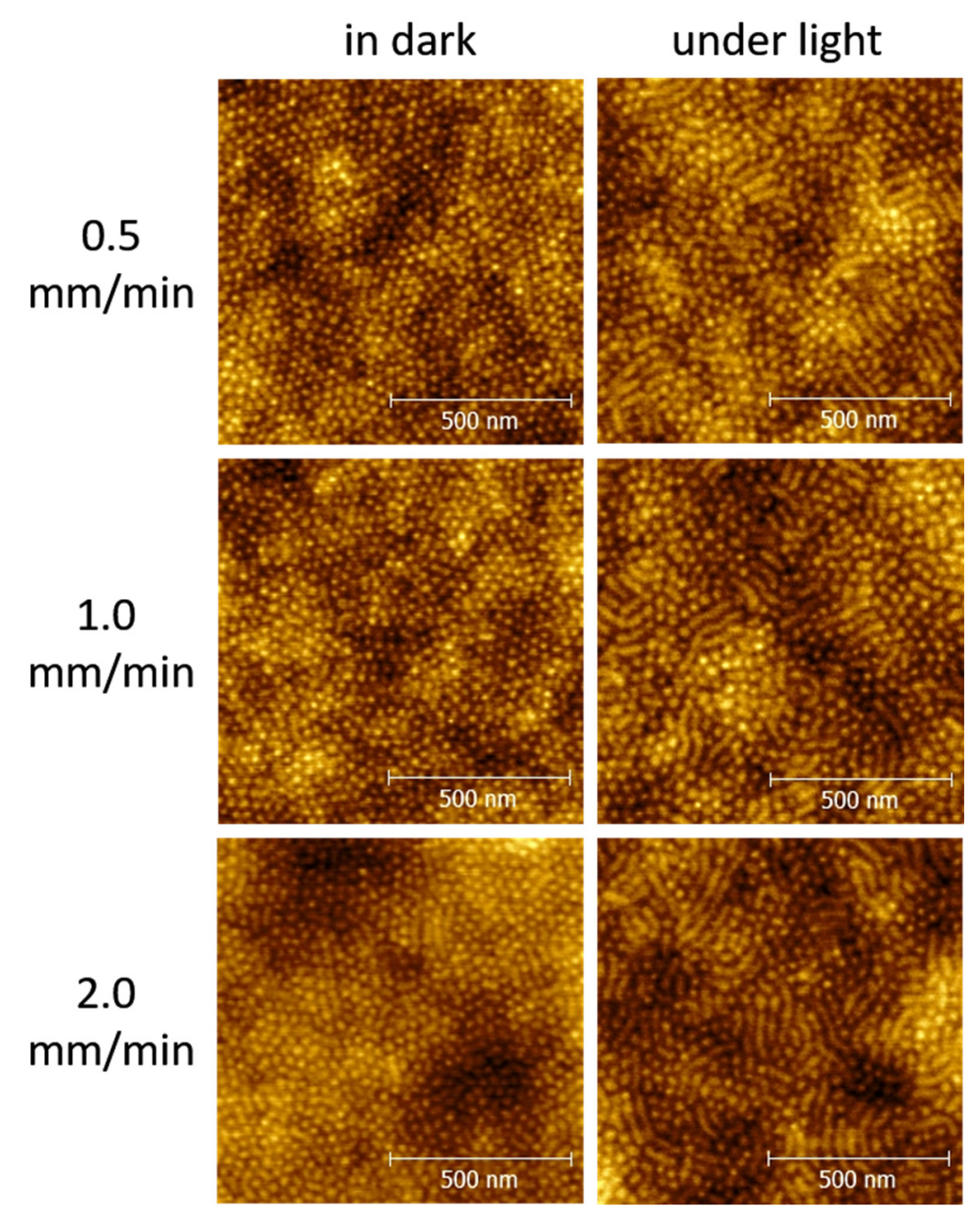

Figure 4. Representative AFM height images of films dip-coated in the dark and under LED illumination from a THF solution of $\mathrm{P} / \mathrm{CHAB}_{0.25}$ at the withdrawal rates indicated.

The dots at the low dip-coating rates are interpreted as spheres because of the very low $\mathrm{BHAB}$ content involved, supported by previous results for similar films containing NCOOH for which cross-sectional transmission electron micrographs combined with AFM surface patterns of dots indicated a spherical morphology. ${ }^{30}$ In contrast, at the highest dip-coating rates for the BHAB system, the dots that are present (mixed with stripes) are interpreted as vertical cylinders, since the increase in BHAB uptake ratio has already transformed the morphology into cylindrical (as evidenced by the stripe pattern) and the BHAB content is now at its highest, leaving no reason for the morphology to return to spherical. A reorientation from horizontal to vertical cylinders with an overlap region (mixed dots and stripes), accompanied by increasing film thickness, was also 
observed for the NCOOH system in THF and for the NOH and NCOOH systems in $p$-dioxane, and supported by cross-sectional TEM micrographs. ${ }^{26}$ It may be mentioned that, for dip-coating from higher $\mathrm{BHAB}$ molar ratio solutions $\left(\mathrm{P} / \mathrm{BHAB}_{0.50}\right.$ and $\left.\mathrm{P} / \mathrm{BHAB}{ }_{1.0}\right)$, stripes are obtained already at the lowest dip-coating rate used, as expected for the BHAB contents involved (see Figure $\mathrm{S} 2$ for the BHAB uptake ratios as a function of dip-coating rate).

It should be added here that the dip-coated films of pure $\mathrm{P}$ give a dot pattern over the entire dip-coating rate range (Table 2 and Figure S1a), except for the appearance as well of some very short stripes in the draining regime. This had not been observed for pure PS-P4VP of somewhat higher molecular weight but similar P4VP content (refs 25 and 26 and Section S3 of the SI). Possibly, this reflects the beginning of transitioning into the cylindrical morphology, which could be related to the difference in micellar character of the two solutions and/or to greater molecular mobility of the lower molecular weight chains that allows the morphology to evolve further along the kinetic pathway towards the bulk equilibrium morphology (the latter being close to the cylindrical/lamellar boundary ${ }^{39}$ ) before immobility sets in. In this case, the greater fraction of dots in the image for $80 \mathrm{~mm} / \mathrm{min}$ could result from vertically oriented cylinders, particularly if the cross-sectional solvent gradient in the drying film combined with its thickness tend to favor such an orientation. ${ }^{40,41}$

The CHAB-containing films without illumination start with a dot pattern for the lowest dip-coating rates $(0.5,1$ and $2 \mathrm{~mm} / \mathrm{min})$, then show a mixed dot-stripe pattern at $4 \mathrm{~mm} / \mathrm{min}$ (compared to $1 \mathrm{~mm} / \mathrm{min}$ for BHAB) and stripes only for $7 \mathrm{~mm} / \mathrm{min}$ (compared to $2 \mathrm{~mm} / \mathrm{min}$ for BHAB) (Table 2, Figures 4 and S1d). In other words, the spherical/horizontal cylindrical morphology transition occurs at a higher dip-coating rate for $\mathrm{CHAB}$ than for $\mathrm{BHAB}$, corresponding to a measured uptake ratio of approximately 0.2 for $\mathrm{CHAB}$ (compared to approximately 0.1 for BHAB). This indicates that roughly double the amount of CHAB compared to BHAB is necessary for the morphology transition to occur. Since the computed hard-core (occupied) volumes of BHAB and $\mathrm{CHAB}$ are similar (172 and $178 \mathrm{~cm}^{3} / \mathrm{mol}$, respectively), the smaller amount of BHAB needed can be rationalized by its flexible $n$-butyl tail contributing to greater free volume in the $\mathrm{P} 4 \mathrm{VP}+\mathrm{BHAB}$ phase as well as greater plasticization of this phase allowing further evolution of the morphology before molecular immobility sets in. For comparison, the uptake ratio threshold for the same transition was found to be about 0.5 for $\mathrm{NCOOH}$, consistent with it being a smaller and more rigid molecule than $\mathrm{BHAB} .{ }^{31}$ 
In the draining regime, a greater fraction of dots are observed for the CHAB system compared to the BHAB system: a few are apparent already at $20 \mathrm{~mm} / \mathrm{min}$ and they dominate (with a minor amount of short stripes) at $80 \mathrm{~mm} / \mathrm{min}$. Again, these dots are interpreted as resulting from vertically oriented cylinders. The fact that the film thicknesses and uptake ratios in this region are very similar for the two systems suggests that the change from horizontal to vertical cylinders occurs at somewhat lower film thicknesses in the presence of CHAB compared to BHAB. Knowing that interfacial interactions with the substrate (or wetting layer) can influence the orientation of cylindrical BCP morphologies in thin films, ${ }^{42}$ a possible explanation is that the much more polar $\mathrm{CHAB}$ contributes in such a way to the attributes of the wetting layer, which is composed of P4VP presumably mixed with some SM, that it better promotes vertical cylindrical orientation than does BHAB.

With illumination. The effect of illumination on the average film thicknesses and uptake ratios in the capillary and draining regimes are shown by the open symbols in Figure 2. Overall, the changes observed are similar for the BHAB and CHAB systems. For both, illumination, while leaving the V-shape of the thickness curves intact (Figure 2a), approximately doubles the film thicknesses in the capillary regime (i.e. for dip-coating rates of $4 \mathrm{~mm} / \mathrm{min}$ and less). The effect of illumination on thickness then decreases rapidly for 7 and $10 \mathrm{~mm} / \mathrm{min}$, and is reduced to less than $20 \%$ in the draining regime. Since a very similar trend was found for SM-free solutions of P in THF (Figure 2a), this effect of illumination cannot be related to the azo groups. Instead, we attribute the thickness increase to optical heating of the substrate and thus of the solvent in the meniscus during dip-coating. It is known that the film thickness in the capillary regime increases with the temperature of the dip-coating solution through its effect on the solvent evaporation rate that dominates this regime. ${ }^{24}$ In the draining regime, the film thickness is influenced little if any by temperature according to ref 24 . In the present case, while the increase is much less than in the capillary regime, there appears to be some effect. If real, we speculate that the high volatility of THF (compared to $\mathrm{H}_{2} \mathrm{O}$ used in ref 24) may exert a role even in the draining regime in connection with the surrounding atmosphere, whose saturation level (THF partial pressure) is not controlled. The same trends as for THF were observed when comparing dip-coating in the dark and under illumination from solutions of chloroform (Figure S3), which has a slightly higher evaporation rate than THF (vapor pressures of 26.2 and $21.6 \mathrm{kPa}$, respectively, at $25^{\circ} \mathrm{C}^{43}$ ). 
Figure $2 \mathrm{~b}$ shows that illumination increases the uptake ratio of BHAB and $\mathrm{CHAB}$ to a similar extent over the entire dip-coating rate range investigated. To rationalize this increase under illumination, we had previously speculated (for the capillary regime) that the increased dipole moment of BHAB in the cis compared to trans state $(4.92$ vs. $1.74 \mathrm{D}$, according to our refined DFT calculations) could increase the electrostatic affinity of BHAB with P4VP under illumination. ${ }^{28}$ This increased affinity would reduce the washing out effect in the capillary regime, analogous to the higher uptake ratio observed in this regime when dip-coating with solvents that do not compete with the SM-VP H-bond. ${ }^{26}$ However, computations do not support the hypothesis that the P4VP-BHAB hydrogen-bonding strength increases upon photoisomerization, since the difference is only $0.2 \mathrm{~kJ} / \mathrm{mol}$ (trans-BHAB-ethylpyridine and cis-BHAB-ethylpyridine interaction strengths are -49.0 and $-49.2 \mathrm{~kJ} / \mathrm{mol}$, respectively). It is likewise not supported by IR spectroscopy, which showed no change in the position of the H-bonded P4VP band at $1009 \mathrm{~cm}^{-1}$ upon isomerizing more than $90 \%$ of the BHAB molecules into cis conformation. Moreover, the much higher dipole moment of CHAB in the trans state $(8.49 \mathrm{D})$, without a significant increase in uptake ratio compared to $\mathrm{BHAB}$ in the trans state, and the smaller cis-CHAB dipole moment (7.02 D) as compared to trans-CHAB, still leading to a photoinduced increase in uptake ratio, are inconsistent with the hypothesis. It is also highly improbable that azo-azo interactions or crystallization play a role given the low solution azo/VP molar ratio of 0.25 .

An alternative explanation for the photoinduced increase in uptake ratio, at least in the capillary regime, is again the faster solvent evaporation rate caused by the above-mentioned heating effect, leaving less time for small-molecule extraction back into solution. Illuminationinduced heating leading to a faster solvent evaporation rate and thus thicker films and higher uptake ratios in the capillary regime is supported by varying the light intensity (related to the heating power), as shown in Figure $\mathrm{S} 4$ for the $\mathrm{P} / \mathrm{BHAB}_{0.25}$ system at a dip-coating rate of 0.5 $\mathrm{mm} / \mathrm{min}$. It is less clear why the uptake ratio also increases in the draining regime under illumination. Possibly it is related to the capacity of the fluid that drains back into solution during dip-coating to extract a fraction of the small molecule content; i.e., the amount of fluid that drains back should be reduced by a faster evaporation rate caused by increased temperature, thereby resulting in less extraction. Such an effect could also explain why the SM uptake in the draining regime did not quite reach the solution ratio (Figure 2b). Moreover, if less fluid is drained, this 
could also rationalize the apparent small increase in thickness of the films dip-coated under illumination (Figure 2a).

As described in ref 28, dip-coating from the $\mathrm{P} / \mathrm{BHAB}_{0.25}$ solution under illumination (Table 2, Figures 3 and S1c) leads to films characterized by a surface pattern of stripes at $0.5 \mathrm{~mm} / \mathrm{min}$ already, indicating that illumination causes the spherical/cylindrical morphology transition to move to lower dip-coating rates. For the analogous CHAB system (Table 2, Figures 4 and S1e), stripes are similarly observed starting at the lowest dip-coating rates used, although this time they are mixed with dots. These dots may reflect spheres, in which case, while illumination clearly moves the morphology transition to lower rates like in the BHAB system, the effect is less pronounced in that it prolongs the mixed morphology region to lower dip-coating rates without becoming fully cylindrical in the range investigated. The low SM uptake at these rates, which is comparable to that in the dark at $2 \mathrm{~mm} / \mathrm{min}$ and for which the morphology is still spherical supports the assignment of dots to spheres. On the other hand, given the much shorter cis lifetime for CHAB (Table 1), which implies greater trans-cis-trans cycling that likely increases the free volume in the system (see next paragraph), and the film thicknesses involved, perhaps along with the indication above (in the draining regime in the dark) that $\mathrm{CHAB}$ appears to promote vertical orientation of cylindrical phases more readily than BHAB, it cannot be ruled out that the dots may also be due to vertically oriented cylinders. It may be added that illumination appears to have little effect on the dip-coating rate where cylindrical reorientation begins to occur in the draining regime for either system.

The photoinduced shift of the spherical/cylindrical morphology transition to lower dipcoating rates for both the BHAB and $\mathrm{CHAB}$ systems is clearly caused, at least in part, by the photoinduced increase in azobenzene uptake. Another contribution comes from the greater free volume associated with the more globular shape of the cis isomers (which are present to more than 90\% under illumination in both cases; Table 1) compared to the more rod-like trans isomers, as well as the increase in free volume caused by trans-cis-trans cycling. Taking into account that the higher number of trans-cis-trans isomerization events per unit time under similar illumination conditions for $\mathrm{CHAB}$ compared to $\mathrm{BHAB}$, it is expected that $\mathrm{CHAB}$ generates more motion and greater free volume in the material, and thus should be more efficient than BHAB at moving the dot-to-stripe transition to lower dip-coating rates compared to in the dark. However, there may be compensating factors that play a role. For example, the flexible BHAB $n$-butyl tail, to which the 
lower dip-coating dot-stripe transition rate of $\mathrm{BHAB}$ compared to $\mathrm{CHAB}$ was attributed (which must also be taken into account), may contribute to still greater free volume in the cis state as well as to a greater plasticization effect in the drying film, thus retarding the point where molecular immobility sets in and consequently allowing somewhat greater evolution of the morphology before it is frozen in.

The above observations for $\mathrm{P} / \mathrm{BHAB}_{0.25}$ comparing film thickness, uptake ratio and surface pattern as a function of dip-coating rate in the dark and under illumination were comparable for a PS-P4VP ( $\left.\mathrm{P}^{\prime}\right)$ of very similar P4VP volume fraction but $\sim 60 \%$ higher molecular weight $(36.5 \mathrm{k}-$ $16 \mathrm{k}$ vs. the above $24 \mathrm{k}-9.5 \mathrm{k}$ ), as shown and commented on in Section S3 of the Supporting Information. The higher molecular weight reduces the solubility of the P4VP block in THF, resulting in a soft micellar solution. ${ }^{26,30}$ The most significant differences between the $\mathrm{P}^{\prime}$ and $\mathrm{P}$ systems are that the surface morphology transition in the dark occurs at a slightly higher dipcoating rate (near $2 \mathrm{~mm} / \mathrm{min}$ for $\mathrm{P}^{\prime}$ instead of $1 \mathrm{~mm} / \mathrm{min}$ for $\mathrm{P}$ ) and, under illumination, the $\mathrm{P}^{\prime}$ films show a mixed dot-and-stripe pattern rather than a pure stripe pattern for the dip-coating rates below $2 \mathrm{~mm} / \mathrm{min}$. These effects on the dip-coated films are attributed to the solution micellar structure and/or molecular weight-related viscosity changes (see Section S3).

It must be emphasized that the photoinduced morphology changes occur during the dipcoating film formation procedure. We have shown previously, ${ }^{28}$ by illuminating a dry dotpatterned BHAB-containing film of the same composition as above and dip-coated in the dark at $0.5 \mathrm{~mm} / \mathrm{min}$, that it is not possible to induce a change in surface pattern by post-exposure to light because of the high block $\mathrm{T}_{\mathrm{gS}}$ in the dry state that prevents sufficient molecular mobility. Furthermore, the in situ pattern formation process implies that what is observed on the film surface should reflect the morphology within the film, particularly in the capillary regime where the film builds up gradually through capillary feeding while being continuously illuminated.

Effects of Solvent (Toluene vs. THF). By changing the solvent from THF to toluene, several potentially important characteristics are modified. First, toluene has a much lower evaporation rate, which is especially significant for the capillary regime and consequent film thicknesses. ${ }^{26}$ Second, toluene is not a hydrogen-bond competitor for the azo-VP H-bond in solution, which affects the uptake ratio in the capillary regime. ${ }^{26}$ Third, it leads to a micellar solution (Table S1), where the micelle cores are much harder than in THF (for higher molecular 
weight PS-P4VP). Since the glassy micelles are essentially immobile, they lead to little if any morphology evolution during solvent evaporation from the drying film. ${ }^{26}$ Here, dip-coated films of $\mathrm{P} / \mathrm{BHAB}$ in toluene are compared with those described above in THF.

Without illumination. For films dip-coated in the dark from a $\mathrm{P} / \mathrm{BHAB}_{0.25}$ toluene solution, no morphology transition was found as a function of withdrawal rate: all films were characterized by a spherical morphology, as were those dip-coated from a BHAB-free toluene solution (Figure S1f and ref 26 for a toluene solution of a similar SM-free P). On the other hand, a higher chromophore molar ratio of 0.50 in toluene induced a dot-stripe transition at slow dip-coating rates (Figure 5 and $\mathrm{S} 1 \mathrm{~g}$ ), as observed for $\mathrm{P} / \mathrm{BHAB}_{0.25}$ in THF; notably, there are predominantly dots (with a small amount of very short stripes) at 0.3 and $0.5 \mathrm{~mm} / \mathrm{min}$ and mainly (short) stripes with a few dots at 1.0 and $2.0 \mathrm{~mm} / \mathrm{min}$. At $3 \mathrm{~mm} / \mathrm{min}$, the surface pattern is unclear, whereas at dipcoating rates of $10-80 \mathrm{~mm} / \mathrm{min}$, it is in the form of dots.

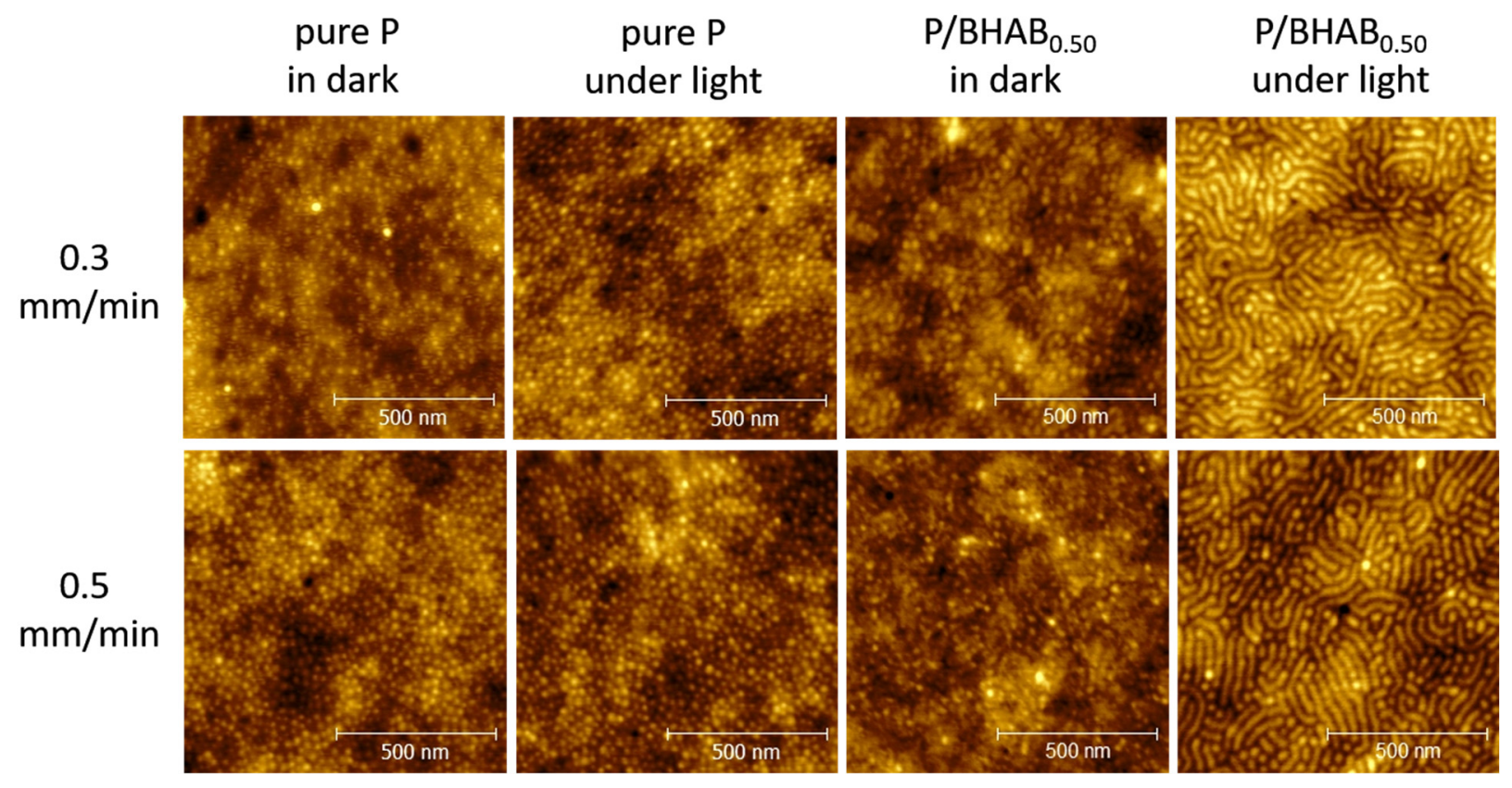

Figure 5. AFM height images of films dip-coated in the dark and under LED illumination from a toluene solution of $\mathrm{P} / \mathrm{BHAB}_{0.50}$, in comparison with a pure $\mathrm{P}$ toluene solution, at the withdrawal rates indicated. 
The corresponding film thicknesses as a function of dip-coating rate, shown in Figure 6a, are significantly thinner in the capillary regime, with the V-minimum displaced to a lower dipcoating rate ( 3 vs. $10 \mathrm{~mm} / \mathrm{min}$ ) and reaching a lower thickness (about 15 vs. $20 \mathrm{~nm}$ ), for toluene solutions compared to THF solutions (see direct comparison in Figure S5a). This is caused by the shift of the capillary regime to lower dip-coating rates due to the slower evaporation rate of toluene. ${ }^{26}$ In parallel, Figures $6 \mathrm{~b}$ and $\mathrm{S} 5 \mathrm{~b}$ show that the BHAB/VP uptake ratio (relative to the solution ratio) starts out higher at low dip-coating rates for toluene compared to THF solutions, attributed to the fact that toluene is not a competitor for the BHAB-P4VP H-bond like THF is, thus decreasing its capacity to wash out $\mathrm{BHAB}$ from the depositing film during its time of contact with the solution meniscus. ${ }^{26}$ In the draining regime, the uptake ratio is close to that in solution for toluene like for THF, as observed previously. ${ }^{26}$

The presence of stripes for the lower dip-coating rates indicates that the cylindrical phase has been reached for $\mathrm{P}_{\mathrm{BHAB}} .5$ in toluene. Since the $\mathrm{SM} / \mathrm{VP}$ ratio is higher at the higher dipcoating rates, the dots observed for those are interpreted as vertically oriented cylinders. This is supported by what was observed previously for dioxane solutions of (equimolar) NOH- and $\mathrm{NCOOH}$-containing films, which also showed dots on the surface of films dip-coated in the draining regime and for which cross-sectional TEM micrographs clearly indicated vertical cylinders. ${ }^{26}$ The very low thickness of the films obtained at the V-minimum of $3 \mathrm{~mm} / \mathrm{min}$, which is about the thickness of the collapsed (dried) brush layer film that shows no particular surface pattern, would explain the unclear surface pattern, which can be interpreted as a relatively featureless surface, for this film. The dots in the films obtained at the two lowest dip-coating rates may be interpreted as spheres or as vertical cylinders, with the results under illumination being supportive of their being spheres (see below). 

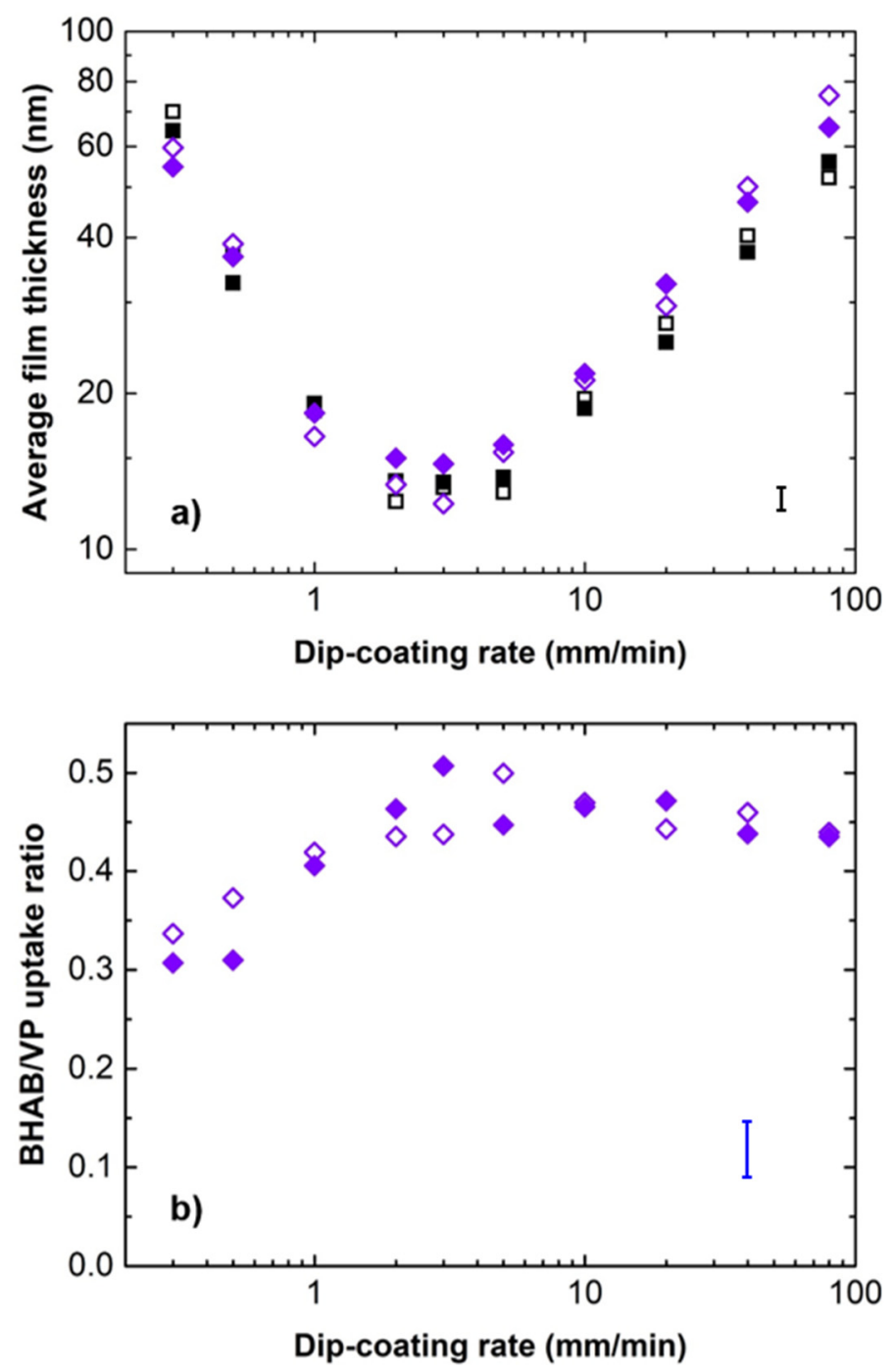

Figure 6. (a) Average film thickness and (b) BHAB/VP uptake ratio as a function of withdrawal rate for films dip-coated in the dark (closed symbols) and under illumination (open symbols) from toluene solutions of $\mathrm{P} / \mathrm{BHAB}_{0.5}$ (violet diamonds) and, in (a) pure $\mathrm{P}$ (black squares). Error bars are indicated in the lower right corners of the graphs.

The need for higher $\mathrm{BHAB}$ content, and thus a higher volume ratio of the $\mathrm{P} 4 \mathrm{VP}+\mathrm{BHAB}$ phase relative to the PS phase, to obtain the cylindrical morphology in films dip-coated from toluene solutions compared to the above THF solutions can be related to the greater immobility of the micelle cores in toluene that slows down the kinetics of phase evolution during solvent evaporation from the dip-coated film. Furthermore, reaching the cylindrical phase for the 0.5 
$\mathrm{BHAB} / \mathrm{VP}$ molar ratio solution contrasts with what was observed for equimolar SM/VP content with $\mathrm{NOH}-$ and $\mathrm{NCOOH}$-containing PS-P4VP solutions in toluene, for which only spherical morphologies were obtained over the whole dip-coating range. ${ }^{26}$ This can be rationalized by the greater occupied volume of BHAB (estimated to be about 1.4 times more than that of $\mathrm{NCOOH}$ ) as well as the larger free volume associated with the more flexible BHAB, particularly its butyl tail. Thus, the total (occupied and free) volume added to the P4VP+SM phase in the film may be roughly doubled when the SM is $\mathrm{BHAB}$ compared to $\mathrm{NCOOH}$ or $\mathrm{NOH}$, which is consistent with the observation made above concerning the amount of SM uptake needed to induce this phase transition for dip-coating in THF solutions (0.1 BHAB/VP vs. 0.5 NCOOH/VP). In addition, the BHAB butyl tail likely exerts a plasticizing effect that confers somewhat greater mobility to the micelle cores in toluene than is possible with the rigid $\mathrm{NCOOH}$ or $\mathrm{NOH}$ molecules, which can allow somewhat greater morphology evolution in the BHAB-containing films during toluene evaporation and before immobility sets in.

With illumination. The average film thicknesses (Figure 6a) and the BHAB/VP uptake ratios (Figure 6b) for dip-coating under illumination from the $\mathrm{P} / \mathrm{BHAB}_{0.50}$ toluene solution are essentially the same as those for dip-coating in the dark, in contrast to what was observed for the THF solution. This can be related to the much lower evaporation rate of toluene (vapor pressure of $3.79 \mathrm{kPa}$ at $25^{\circ} \mathrm{C}$ ) that remains low even in the presence of photoinduced substrate heating so that no thickness increase beyond experimental uncertainty occurs. The surface patterns (Figures 5 and $\mathrm{S} 1 \mathrm{~h}$ ), however, undergo some change under illumination, namely that stripes along with dots are present already at 0.3 and $0.5 \mathrm{~mm} / \mathrm{min}$ dip-coating rates, indicating a similar effect of illumination for these films as for those dip-coated from THF solution. (Figure 5 also shows that there is no effect of illumination on the surface pattern of pure P films, as expected from the absence of a photoresponsive element.) The similarity of toluene to THF regarding the effect of illumination on the surface pattern supports the interpretation of the dots for these rates in toluene in the dark as spheres, since there is no particular reason, especially in the absence of a heating effect, for illumination to cause more horizontally oriented and fewer vertically oriented cylinders. Moreover, although illumination does not change the BHAB/VP uptake ratio, it causes photoisomerization of BHAB (again, more than $90 \%$ cis isomers in the photostationary state; Table 1), which increases the effective $\mathrm{P} 4 \mathrm{VP}+\mathrm{BHAB}$ phase volume fraction, thus rationalizing a change from spherical to cylindrical morphology under illumination. An additional contribution may arise 
from increased free volume due to trans-cis-trans cycling, given that the half-life of the BHAB cis state in the presence of $\mathrm{P}$ in toluene is much faster than in THF (Table 1). At the V-minimum (3 $\mathrm{mm} / \mathrm{min}$ ) under illumination, there are hints of stripes, whereas at higher dip-coating rates, the phase images (which are clearer than the height images in these cases, Figure S1h) show dots, along with very short stripes, indicating a small effect of illumination on the pattern. In summary, the effect of illumination on the morphology for toluene solutions is related to BHAB photoisomerization alone, i.e. to the effective increase in the $\mathrm{P} 4 \mathrm{VP}+\mathrm{BHAB}$ volume fraction arising from the presence of cis isomers and trans-cis-trans cycling, in contrast to the combined effects of photoisomerization and higher uptake ratio for THF solutions.

Summary of Results. It is useful now to summarize the various results obtained, first those in the dark and then those under illumination. In the dark, the thickness and SM uptake tendencies as a function of dip-coating withdrawal rate in PS-P4VP films confirm previous studies with nonazo SMs showing (a) that there is a general V-shaped dependence of the film thickness that delimits the "capillary" and "draining" regimes of dip-coating and (b) that the SM uptake, which is low at slow dip-coating rates in THF, a hydrogen-bond acceptor like P4VP, and moderate in toluene that does not H-bond with the SM, increases with dip-coating rate to near the solution SM/VP ratio. In parallel, changes in the surface pattern take place as a function of dip-coating rate in the systems investigated. Focusing on the best-defined changes between dots and stripes due to spheres and horizontal cylinders in the capillary regime, it was concluded for dip-coating from PS-P4VP/SM non-micellar solutions in THF that greater free volume (as provided by the $n$-butyl tail compared to the cyano tail for the azo SM, BHAB vs. $\mathrm{CHAB}$ ), which adds to the effective $\mathrm{P} 4 \mathrm{VP}+\mathrm{SM}$ volume fraction in the (drying) film, is responsible for displacing the dot-stripe transition to lower dipcoating rates without affecting the film thickness and SM uptake tendencies. A higher PS-P4VP molecular weight (same P4VP content) and/or its soft micellar character displaces the transition to a slightly higher dip-coating rate due to slightly higher solution viscosity that slows down molecular mobility during solvent evaporation. In toluene, where the block copolymer forms a hard-core micellar solution, double the SM content in solution is required to observe the same phase transition as in THF.

For each of these systems, illumination $(365 \mathrm{~nm})$ displaces the dot-stripe transition in the capillary regime to lower dip-coating rates. It also significantly increases the film thickness in the capillary regime and the SM uptake in both the capillary and draining regimes for dip-coating from 
THF solutions, and this to the same extent for BHAB- and CHAB-containing solutions. In contrast, no change in either film thickness or SM uptake is observed for dip-coating from toluene solutions. For THF, the increase in the capillary regime is related to optical heating of the substrate, which affects two dominant aspects of the capillary regime; notably, it increases the solvent evaporation rate as the film forms, and therefore the film thickness, and it reduces the time for SM extraction from the forming film during its contact with the solution, thereby leaving more SM in the film. This effect is not visible for toluene solutions, attributed to its much lower evaporation rate. The photoinduced surface pattern changes can be interpreted in the light of the SM uptake along with knowledge of the SM photochemical and molecular characteristics, in particular in relation to their effects on the effective volume fraction of the $\mathrm{P} 4 \mathrm{VP}+\mathrm{SM}$ phase. In all of the systems, the high cis content in the photostationary state increases this fraction. For THF solutions, the photoinduced increase in SM uptake further increases this fraction. The shorter cis lifetimes of the CHABcontaining THF solution and the BHAB-containing toluene solution (shorter for the former than the latter), compared to the BHAB-containing THF solution, leads to additional free volume due to trans-cis-trans cycling. On the other hand, photoinduced increases in the effective P4VP+SM volume fraction that lower the dip-coating rate at which a morphology change occurs can be countered by anything that decreases the molecular mobility, thereby leading to freezing in of the film morphology at earlier stages of its evolution from the initial solution state towards the equilibrium morphology in the bulk as the solvent evaporates from the film. Thus, higher polymer molecular weight, greater micelle core hardness, and less effective plasticization by the SM (e.g. the cyano tail in $\mathrm{CHAB}$ is less effective compared to the butyl tail in BHAB) can all contribute to reduced molecular mobility.

Thus, a set of design guidelines has been established that should prove useful for eventual applications. Other guidelines can be added in future studies by, for example, modifying the P4VP content, which, if much higher, might allow photocontrol involving lamellar or even inverted cylindrical morphologies, or by using other solvents such as $\mathrm{CHCl}_{3}$, which is not block-selective and thus promotes lamellar morphologies for the BCP used here. ${ }^{26}$ Photoactive molecules with a greater volume difference between their relaxed and photoexcited states can be expected to enhance photoinduced morphology changes. Eventual applications may involve, for example, partial masking of the impinging light so that, in the critical region of dip-coating where the morphology is different in the dark and under illumination, (complex) spatial patterns of the two 
morphologies may be developed. If desirable, it is also easy to subsequently remove the azo SMs with a selective solvent. In general, dip-coating does not lead to long-range lateral order in BCP films, which can be advantageous for certain applications such as broadband antireflective coatings. ${ }^{44,45}$ When needed, long-range order might be achievable by solvent or thermal annealing under illumination or by combining photocontrolled dip-coating with other tools, such as using prepatterned (by chemical or mechanical means) substrates for directed assembly.

\section{CONCLUSIONS}

In this study, we have reached a good understanding of various parameters that affect the in situ control by light of the surface pattern, thickness and SM uptake in dip-coated thin films of supramolecular azo block copolymers where the azo SM derivative H-bonds selectively to one of the blocks. The various aspects, as highlighted in the above summary, constitute guidelines for the design of thin-film materials of interest for in situ manipulation by light during dip-coating. In particular, we showed the importance of the choice of azo SM relative to its photochemical and molecular characteristics (steady-state cis content, cis lifetime, flexible motifs contributing to free volume and plasticization) and of the choice of solvent relative to its vapor pressure, to its interaction with the SM (if competitive or not with the polymer) and its block selectivity (if provoking micellar solutions and affecting core hardness). To this may be added the block molecular weights, both relative (which affects the film morphologies) and absolute (which affects solution viscosity and therefore molecular mobility in the drying film and which can affect micellization). Interestingly, depending on solvent and azo content in solution, LED illumination during dip-coating allows simultaneous changes in the surface pattern, functional SM content and thickness, or in only the SM content and thickness, or in only the thickness. The simplicity of the technique used for the light control, i.e. coupling of an LED to a dip-coating setup, and the

supramolecular character of the materials renders implementation of the technique and design guidelines facile and easily tunable, where any component (block copolymer, azo SM, illumination wavelength, solvent used, etc.) can be changed with minimal effort and cost. 


\section{ASSOCIATED CONTENT}

\section{Supporting Information}

The Supporting Information is available free of charge on the ACS Publications website at DOI:

Representative AFM images, additional tables and figures, results obtained with a higher molecular weight block copolymer (PDF)

\section{AUTHOR INFORMATION}

\section{Corresponding Authors}

*E-mail: c.pellerin@umontreal.ca.

*E-mail: geraldine.bazuin@umontreal.ca.

\section{ORCID}

Jaana Vapaavuori: 0000-0002-5923-0789

Arri Priimagi: 0000-0002-5945-9671

Christian Pellerin: 0000-0001-6144-1318

C. Geraldine Bazuin: 0000-0002-0262-1877

\section{Author Contributions}

JV and JG contributed equally to this work.

\section{Notes}

The authors declare no competing financial interest.

\section{ACKNOWLEDGMENTS}

CGB and CP acknowledge funding by the Natural Sciences and Engineering Research Council (NSERC; grants RGPGP/77200073-2014 and RGPIN/04014-2015) of Canada and the Fonds de Recherche du Québec - Nature et Technologies [FRQNT; grant to the Centre de recherche sur les matériaux auto-assemblés (CRMAA/CSACS) of which the Université de Montréal coauthors were members]. JV was supported by postdoctoral fellowships from the Academy of Finland and the Banting program (Canada). AP acknowledges the financial support of the Academy of Finland (decision numbers 277091 and 312628).

\section{REFERENCES}

(1) Bates, C. M.; Maher, M. J.; Janes, D. W.; Ellison, C. J.; Willson, C. G. Block Copolymer Lithography. Macromolecules 2014, 47, 2-12.

(2) Sinturel, C.; Vayer, M.; Morris, M.; Hillmyer, M. A. Solvent Vapor Annealing of Block Polymer Thin Films. Macromolecules 2013, 46, 5399-5415. 
(3) Bang, J.; Jeong, U.; Ryu, D. Y.; Russell, T. P.; Hawker, C. J. Block Copolymer Nanolithography: Translation of Molecular Level Control to Nanoscale Patterns. Adv. Mater. 2009, 21, 4769-4792.

(4) Bigall, N. C.; Nandan, B.; Gowd, E. B.; Horechyy, A.; Eychmuller, A. High-Resolution Metal Nanopatterning by Means of Switchable Block Copolymer Templates. ACS Appl. Mater. Interfaces 2015, 7, 12559-12569.

(5) Ryu, J. H.; Park, S.; Kim, B.; Klaikherd, A.; Russell, T. P.; Thayumanavan, S. Highly Ordered Gold Nanotubes using Thiols at a Cleavable Block Copolymer Interface. J. Am. Chem. Soc. 2009, 131, 9870-9871.

(6) Malmstrom, J.; Travas-Sejdic, J. Block Copolymers for Protein Ordering. J. Appl. Polym. Sci. 2014, 131, 40360.

(7) Hillmyer, M. A., Nanoporous Materials from Block Copolymer Precursors. In Block Copolymers II. Advances in Polymer Science, V., A., Ed. Springer: Berlin, 2005; Vol. 190, pp 137-181.

(8) Ross, C. A.; Berggren, K. K.; Cheng, J. Y.; Jung, Y. S.; Chang, J. B. Three-Dimensional Nanofabrication by Block Copolymer Self-Assembly. Adv. Mater. 2014, 26, 4386-4396.

(9) Bates, F. S.; Fredrickson, G. H. Block Copolymers-Designer Soft Materials. Physics Today 1999, 52, 32-38.

(10) Luo, M.; Epps, T. H. Directed Block Copolymer Thin Film Self-Assembly: Emerging Trends in Nanopattern Fabrication. Macromolecules 2013, 46, 7567-7579.

(11) Kim, H. C.; Park, S. M.; Hinsberg, W. D. Block Copolymer based Nanostructures: Materials, Processes, and Applications to Electronics. Chem. Rev. 2010, 110, 146-177.

(12) Ruiz, R.; Kang, H.; Detcheverry, F. A.; Dobisz, E.; Kercher, D. S.; Albrecht, T. R.; de Pablo, J. J.; Nealey, P. F. Density Multiplication and Improved Lithography by Directed Block Copolymer Assembly. Science 2008, 321, 936-939.

(13) Bita, I.; Yang, J. K.; Jung, Y. S.; Ross, C. A.; Thomas, E. L.; Berggren, K. K. Graphoepitaxy of Self-Assembled Block Copolymers on Two-Dimensional Periodic Patterned Templates. Science 2008, 321, 939-943.

(14) Segalman, R. A. Patterning with Block Copolymer Thin Films. Mater. Sci. Eng. R Rep. 2005, 48, 191-226. 
(15) Hu, H.; Gopinadhan, M.; Osuji, C. O. Directed Self-Assembly of Block Copolymers: a Tutorial Review of Strategies for Enabling Nanotechnology with Soft Matter. Soft Matter 2014, 10, 3867-3889.

(16) Laforgue, A.; Bazuin, C. G.; Prud'homme, R. E. A Study of the Supramolecular Approach in Controlling Diblock Copolymer Nanopatterning and Nanoporosity on Surfaces. Macromolecules 2006, 39, 6473-6482.

(17) ten Brinke, G.; Ruokolainen, J.; Ikkala, O., Supramolecular Materials Based On HydrogenBonded Polymers. In Supramolecular Materials Based On Hydrogen-Bonded Polymers, Springer, Berlin, Heidelberg: 2007; Vol. 207, pp 113-177.

(18) Tung, S.-H.; Kalarickal, N. C.; Mays, J. W.; Xu, T. Hierarchical Assemblies of BlockCopolymer-Based Supramolecules in Thin Films. Macromolecules 2008, 41, 6453-6462.

(19) Vukovic, I.; Punzhin, S.; Vukovic, Z.; Onck, P.; De Hosson, J. T.; ten Brinke, G.; Loos, K. Supramolecular Route to Well-Ordered Metal Nanofoams. ACS Nano 2011, 5, 6339-6348.

(20) Sidorenko, A.; Tokarev, I.; Minko, S.; Stamm, M. Ordered Reactive Nanomembranes/Nanotemplates from Thin Films of Block Copolymer Supramolecular Assembly. J. Am. Chem. Soc. 2003, 125, 12211-12216.

(21) Tokarev, I.; Krenek, R.; Burkov, Y.; Schmeisser, D.; Sidorenko, A.; Minko, S.; Stamm, M. Microphase Separation in Thin Films of Poly(styrene-block-4-vinylpyridine) Copolymer-2-(4‘-Hydroxybenzeneazo)benzoic Acid Assembly. Macromolecules 2005, 38, 507-516.

(22) Ceratti, D. R.; Louis, B.; Paquez, X.; Faustini, M.; Grosso, D. A New Dip Coating Method to Obtain Large-Surface Coatings with a Minimum of Solution. Adv. Mater. 2015, 27, 4958-4962.

(23) Grosso, D. How to Exploit the Full Potential of the Dip-Coating Process to Better Control Film Formation. J. Mater. Chem. 2011, 21, 17033-17038.

(24) Faustini, M.; Louis, B.; Albouy, P. A.; Kuemmel, M.; Grosso, D. Preparation of Sol-Gel Films by Dip-Coating in Extreme Conditions. J. Phys. Chem. C 2010, 114, 7637-7645.

(25) Roland, S.; Prud'homme, R. E.; Bazuin, C. G. Morphology, Thickness, and Composition Evolution in Supramolecular Block Copolymer Films over a Wide Range of Dip-Coating Rates. ACS Macro Lett. 2012, 1, 973-976. 
(26) Roland, S.; Gamys, C. G.; Grosrenaud, J.; Boissé, S.; Pellerin, C.; Prud'homme, R. E.; Bazuin, C. G. Solvent Influence on Thickness, Composition, and Morphology Variation with Dip-Coating Rate in Supramolecular PS-b-P4VP Thin Films. Macromolecules 2015, $48,4823-4834$.

(27) Roland, S.; Pellerin, C.; Bazuin, C. G.; Prud'homme, R. E. Evolution of Small Molecule Content and Morphology with Dip-Coating Rate in Supramolecular PS-P4VP Thin Films. Macromolecules 2012, 45, 7964-7972.

(28) Vapaavuori, J.; Grosrenaud, J.; Pellerin, C.; Bazuin, C. G. In Situ Photocontrol of Block Copolymer Morphology During Dip-Coating of Thin Films. ACS Macro Lett. 2015, 4, 1158-1162.

(29) Li, L.; Gao, P.; Wang, W.; Mullen, K.; Fuchs, H.; Chi, L. Growth of Ultrathin Organic Semiconductor Microstripes with Thickness Control in the Monolayer Precision. Angew. Chem. Int. Ed. 2013, 52, 12530-12535.

(30) Roland, S.; Gaspard, D.; Prud'homme, R. E.; Bazuin, C. G. Morphology Evolution in Slowly Dip-Coated Supramolecular PS-b-P4VP Thin Films. Macromolecules 2012, 45, 5463-5476.

(31) Marenich, A. V.; Cramer, C. J.; Truhlar, D. G. Universal Solvation Model based on Solute Electron Density and on a Continuum Model of the Solvent defined by the Bulk Dielectric Constant and Atomic Surface Tensions. J. Phys. Chem. B 2009, 113, 6378-6396.

(32) Serra, F.; Terentjev, E. M. Effects of Solvent Viscosity and Polarity on the Isomerization of Azobenzene. Macromolecules 2008, 41, 981-986.

(33) Lee, J. N.; Park, C.; Whitesides, G. M. Solvent Compatibility of Poly(dimethylsiloxane)based Microfluidic Devices. Anal. Chem. 2003, 75, 6544-6554.

(34) Joshi, N. K.; Fuyuki, M.; Wada, A. Polarity controlled reaction path and kinetics of thermal cis-to-trans isomerization of 4-aminoazobenzene. J. Phys. Chem. B 2014, 118, 1891-1899.

(35) Garcia-Amorós, J.; Sánchez-Ferrer, A.; Massad, W. A.; Nonell, S.; Velasco, D. Kinetic study of the fast thermal cis-to-trans isomerisation of para-, ortho- and polyhydroxyazobenzenes. Phys. Chem. Chem. Phys. 2010, 12, 13238-13242.

(36) Poutanen, M.; Ahmed, Z.; Rautkari, L.; Ikkala, O.; Priimagi, A. Thermal Isomerization of Hydroxyazobenzenes as a Platform for Vapor Sensing. ACS Macro Lett. 2018, 7, 381-386. 
(37) Devi, S.; Saraswat, M.; Grewal, S.; Venkataramani, S. Evaluation of Substituent Effect in Z-Isomer Stability of Arylazo-1 H-3,5-dimethylpyrazoles: Interplay of Steric, Electronic Effects and Hydrogen Bonding. J. Org. Chem. 2018, 83, 4307-4322.

(38) Garcia-Amorós, J.; Velasco, D. Understanding the Fast Thermal Isomerisation of Azophenols in Glassy and Liquid-Crystalline Polymers. Phys. Chem. Chem. Phys. 2014, 16, 3108-3114.

(39) Ruokolainen, J.; Saariaho, M.; Ikkala, O.; ten Brinke, G.; Thomas, E. L.; Torkkeli, M.; Serimaa, R. Supramolecular Routes to Hierarchical Structures: Comb-Coil Diblock Copolymers Organized with Two Length Scales. Macromolecules 1999, 32, 1152-1158.

(40) Phillip, W. A.; Hillmyer, M. A.; Cussler, E. L. Cylinder Orientation Mechanism in Block Copolymer Thin Films Upon Solvent Evaporation. Macromolecules 2010, 43, 7763-7770.

(41) Morikawa, Y.; Nagano, S.; Watanabe, K.; Kamata, K.; Iyoda, T.; Seki, T. Optical Alignment and Patterning of Nanoscale Microdomains in a Block Copolymer Thin Film. Adv. Mater. 2006, 18, 883-886.

(42) Darling, S. B. Directing the Self-Assembly of Block Copolymers. Prog. Polym. Sci. 2007, 32, 1152-1204.

(43) Haynes, W. M., CRC Handbook of Chemistry and Physics. 95th ed.; CRC Press: Boca Raton, FL, USA, 2014.

(44) Trompoukis, C.; Massiot, I.; Depauw, V.; El Daif, O.; Lee, K.; Dmitriev, A.; Gordon, I.; Mertens, R.; Poortmans, J. Disordered Nanostructures by Hole-Mask Colloidal Lithography for Advanced Light Trapping in Silicon Solar Cells. Opt. Express 2016, 24, A191-201.

(45) Taylor, C. D.; Busse, L. E.; Frantz, J.; Sanghera, J. S.; Aggarwal, I. D.; Poutous, M. K. Angle-of-Incidence Performance of Random Anti-Reflection Structures on Curved Surfaces. Appl. Opt. 2016, 55, 2203-2213. 